\title{
The Nevzorov Airborne Hot-Wire LWC-TWC Probe: Principle of Operation and Performance Characteristics
}

\author{
A. V. Korolev, J. W. Strapp, and G. A. IsaAC \\ Atmospheric Environment Service, Cloud Physics Research Division, Downsview, Ontario, Canada
}

A. N. NeVZOROV

Central Aerological Observatory, Dolgoprudny, Russia

1 July 1997 and 3 December 1997

\begin{abstract}
The Nevzorov liquid water content (LWC) and total water content (TWC) probe is a constant-temperature, hot-wire probe designed for aircraft measurements of the ice and liquid water content of clouds. The probe consists of two separate sensors for measurements of cloud liquid and total (ice plus liquid) water content. Each sensor consists of a collector and a reference winding. The reference sensors are shielded from impact with cloud particles, specifically to provide an automatic compensation for convective heat losses. This results in a potentially improved sensitivity over uncompensated probes such as the King LWC probe. The Nevzorov probe has been used in four Canadian field experiments on the National Research Council (NRC) Convair580 since 1994. Intercomparison of Nevzorov LWC, TWC, King, and two PMS Forward Scattering Spectrometer Probes show good agreement in liquid clouds, although the Nevzorov probe displays distinct advantages in low-LWC situations due to a more stable baseline. The sensitivity of the probe is estimated to be approximately $0.003-$ $0.005 \mathrm{~g} \mathrm{~m}^{-3}$. The accuracy of LWC measurements in nonprecipitating liquid clouds is estimated as $10 \%-15 \%$. Tests at the NRC high-speed icing tunnel have provided verification of the TWC measurement for small frozen droplets to an accuracy of approximately $10 \%-20 \%$, but verification in snow and natural ice crystals has not yet been possible due to the absence of any accurate standards. The TWC measurement offers not only the possibility of direct measurements of ice content but also improved liquid water contents in drizzle situations. Airborne measurements have provided data on the baseline drift and sensitivity of the probe and have provided comparisons to other conventional instruments. Several cases have been documented that exhibit the unique capabilities of the instrument to separate the ice and liquid components of supercooled clouds.
\end{abstract}

\section{Introduction}

Cloud water content is one of the most fundamental measurements in cloud physics. Many research organizations currently use hot-wire probes for airborne measurements of liquid water content (LWC). The most widely used hot-wire devices during the past two decades have been the Johnson-Williams probe and, more recently, the Commonwealth and Industrial Research Organisation (CSIRO) Particle Measuring Systems (PMS) King probe (King et al. 1978). Estimates of the total water content (TWC: ice plus liquid water content) have been accomplished mainly from the interpretation of particle image data or from prototype instruments that evaporate all hydrometeors and measure humidity changes (Brown 1993; Twohy et al. 1997). Real-time information on cloud ice water content (IWC) has been difficult to achieve.

Corresponding author address: Dr. Alexei V. Korolev, Atmospheric Environment Service, Cloud Physics Research Division, 4905 Dufferin Street, Downsview, ON M3H 5T4, Canada.

E-mail: akorolev@rpn.aes.doe.ca
The Nevzorov LWC-TWC probe is a constant-temperature, hot-wire probe designed for aircraft measurements of the ice and liquid water content of clouds and fogs. The first version of this probe [originally named "Izmeritel Vodnosti Oblakov" (IVO) for Cloud Water Content Meter] was developed in the Cloud Physics Laboratory of the Russian Central Aerological Observatory in the mid-1970s. The probe was designed to make measurements of total water content (Nevzorov 1980, 1983). A second version added a sensor designed to be sensitive to liquid particles but insensitive to ice particles, thereby providing a device capable of separately measuring both the ice and liquid components of clouds (Nevzorov and Shugaev 1992a).

The Nevzorov LWC-TWC probe, like the PMS King probe, provides a measurement of LWC, which is fully calculable from first principles of the heat transfer on the sensor wire. In this regard, the probe in theory does not require comparison to another device or standard in order to obtain its absolute calibration. The primary sources of error in the LWC calculation are the measurements of the probe sensor area, the estimate of the 
resistance of the sensor wire at its operating temperature, the estimation of the various empirical constants in the heat transfer equations, and the estimation of the collection efficiencies of the wire. It is our contention that the hot-wire technique is fundamentally simpler than techniques employing optical sensors that derive LWC from complicated optical response calculations, often require an independent instrument comparison for absolute calibration, and respond in an unknown manner to mixed-phase situations. The simple hot-wire technique, with the introduction of the Nevzorov probe, is for the first time capable of providing real-time measurements of the phase separation in mixed-phase clouds and phase ratios that are arguably more accurate than those produced by any other combination of conventional probes.

The Nevzorov probe was installed by the Atmospheric Environment Service on the National Research Council (NRC) Convair580 in 1994 and has since been used in six Canadian field experiments. In this paper, the principle of operation of the probe is introduced, performance tests related to the sensitivity of the probe are discussed, and examples of the unique phase separation capabilities are shown. Comparisons are made with other conventional instruments.

\section{Technical description}

\section{a. The sensor configuration}

Figure 1 shows the basic configuration of TWC and LWC sensors. Each sensor consists of a collector wire and a reference wire heated to the same temperature. The temperature is maintained constant with the help of a bridge feedback. The sampling surface of the leading sensor wire, or collector, is exposed to the airflow and cloud particles. The reference wire, or compensation sensor, is designed to be aerodynamically protected from collision with particles and should remain dry in clouds. Both the collector and reference sensors are ventilated by the same airflow, albeit with a different orientation relative to the flow. The reference sensor measurement is used to perform a precise removal of the dry-air, heat-loss term.

The LWC and TWC sensors consist of close singlelayer windings of enamel-covered nickel wire. For the TWC probe, the collector winding is cemented to the hollow cone at the end of a plastic (textolite) cylinder, and the reference sensor is wound within a shallow groove cut into the same cylinder (Fig. 1a). For the LWC probe, both collector and reference sensors are wound on solid copper rods and cemented to the opposite edges of a flat textolite plate.

The diameter of the conical sample area of the TWC collector is $8 \mathrm{~mm}$, and the cone angle is $120^{\circ}$. The cylindrical LWC collector measures $1.8 \mathrm{~mm}$ in diameter by $16 \mathrm{~mm}$ in length. The resistance of the sensor wires at $90^{\circ} \mathrm{C}$ is typically between 2.5 and $3.5 \Omega$.

The phase discrimination capability of the TWC and
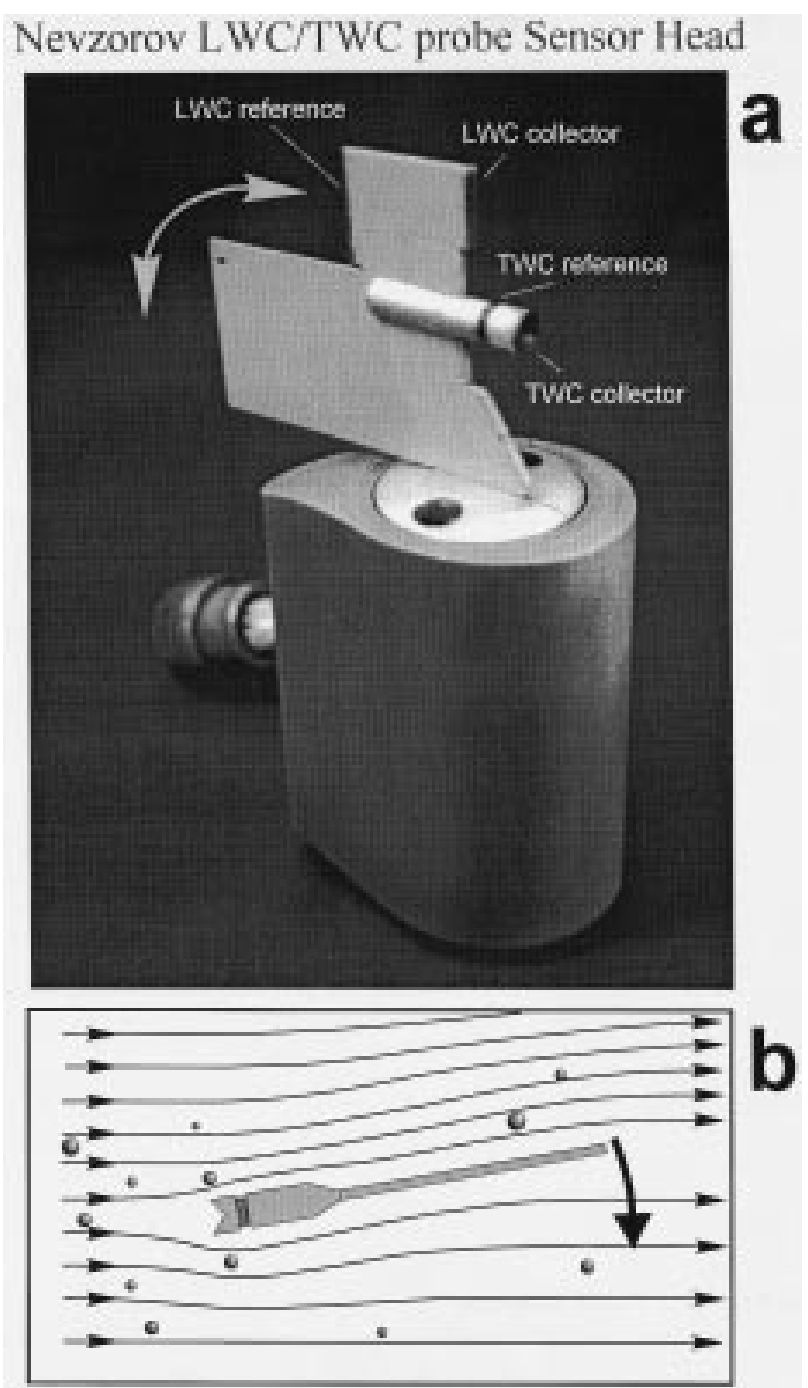

FIG. 1. (a) Photograph of the Nevzorov LWC-TWC sensor head, including the pivoting sensor vane. (b) The self-adjustment of the sensor vane parallel to the airstream.

LWC collectors results from the difference in the behavior of liquid and solid particles impacting with their surfaces. Small liquid droplets, after collision with LWC or TWC collector sensors, should be flattened into a thin surface film and should completely evaporate (Figs. $2 \mathrm{a}, \mathrm{b})$. Ice particles should tend to remain inside the conical hollow of the TWC collector (Fig. 2b) until they melt and evaporate. However, ice particles are expected to instantly break away from the convex surface of the LWC collector (Fig. 2a) with negligible heat expended relative to that for complete ice evaporation. The performance of the Nevzorov LWC sensor in many ways is similar to that of the King probe since the geometrical dimensions of the cylindrically wound wire are similar.

The LWC and TWC sensors are mounted on the same flow-sensitive vane plate (Fig. 1a), which when mounted horizontally is designed to remain parallel to the air- 


\section{PHASE DISCRIMINATION CAPABILITY}
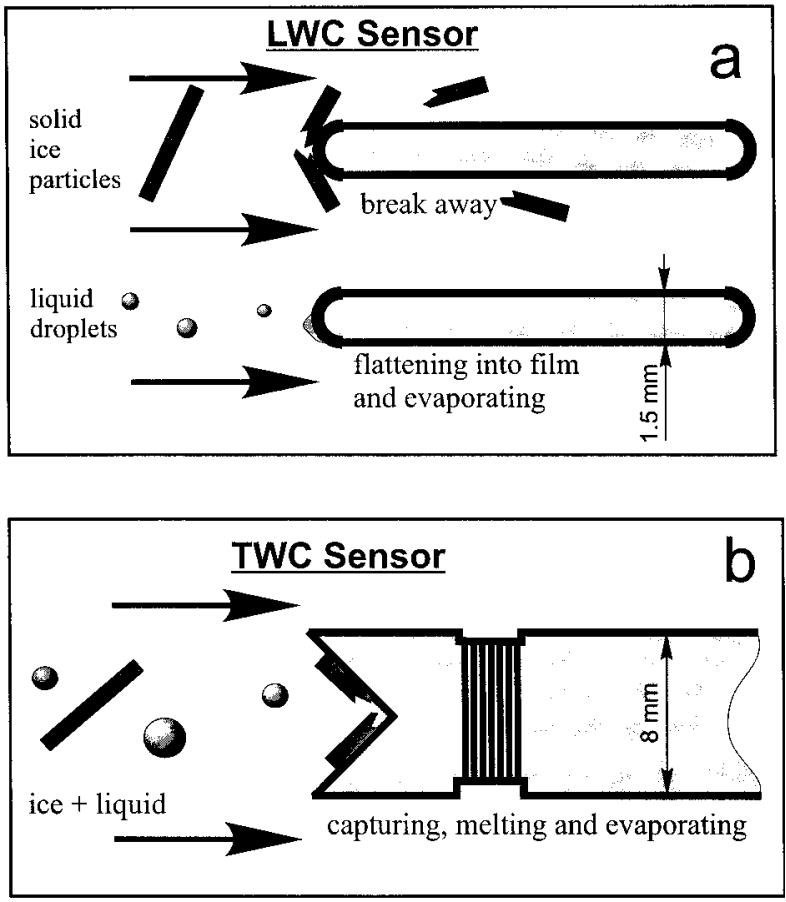

FIG. 2. The principle of phase discrimination for the (a) LWC and (b) TWC sensors.

stream during aircraft pitch changes (Fig. 1b). This pivoting vane stabilizes the flow characteristics of the sensors and further protects the reference sensors from particle impacts.

The vane shown in Figs. 1a and 1b incorporates leading-edge heaters to protect the sensors from the buildup of ice in supercooled clouds. These heaters are effective in light icing situations. A new vane design is currently being tested to improve the anti-icing capability in the moderate to heavy icing situations expected in aircraft icing studies.

\section{b. Electronic circuitry}

A simplified schematic of the control circuitry is presented in Fig. 3. The collector and reference windings form the temperature-dependent arms of two different bridges. The bridges are balanced to a set resistance, which determines the temperature of the active sensor wire. The temperature of the collector and reference sensors are set by the precise adjustable resistors $\mathrm{R}^{\prime}$ and $\mathrm{R}^{\prime \prime}$ (Fig. 3), respectively, that are mounted on the front panel of the control box. The relation between the sensor temperature and the resistance $\mathrm{R}^{\prime}$ (or $\mathrm{R}^{\prime \prime}$ ) is established based on laboratory calibrations.

The reference sensor forms the arm of a resistance bridge $\mathrm{B}$ controlled by alternating current (AC). This bridge consists of the AC amplifier of the imbalance (error) signal (1), the phase-sensitive detector (2), the

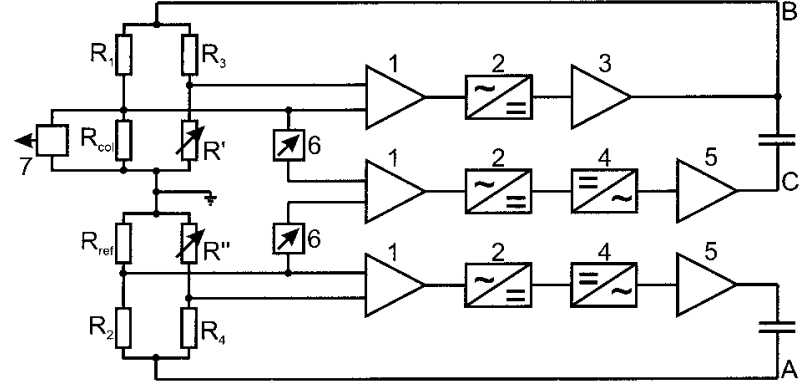

FIG. 3. General electronics schematic of the Nevzorov probe.

amplitude modulator of the gain reinserted AC carrier (4), and the power amplifier loaded by the bridge (5). Other control circuits are built in an analogous way to operate with AC error signals with no effect from the DC component.

The collector bridge is balanced with both AC (C) and $\mathrm{DC}(\mathrm{B})$ in parallel, where the AC voltage is exactly proportional to that across the reference winding, and is adjusted so that the DC is zero while adjusting the collector bridge with AC power only. The adjustment is performed in cloud-free air using potentiometer (6) (Fig. 3). Since the reference winding is aerodynamically protected from the impact with cloud particles, the AC power across the reference sensor inside and outside the cloud should stay the same if temperature, true airspeed (TAS), and pressure do not change. Under these conditions, the AC power across the collector sensor will stay constant since it is designed to be proportional to that across the reference sensor. Thus, the DC circuit of the collector winding is inactive outside of cloud and operates as soon as cloud particles impact and cool the collector winding. The cloud water content signal is carried on the DC component voltage of the collector winding, which is easily measured by suppressing the AC voltage with a low-pass filter (7). Thus, the cooling caused by the dry-air convective heat loss is compensated by the reference winding of the sensor, such that the DC power measured from the collector winding is only that required to evaporate water and ice particles. The water content can then be directly derived from the measured DC power with the appropriate heat balance equation.

\section{c. LWC and TWC calculation}

Since the reference sensor is designed not to impact with cloud particles, its heat losses are due primarily to convection. Thus, the reference sensor bridge is balanced by the power

$$
P_{r}=\frac{V_{r}^{2}}{R_{r}}=\alpha_{r}\left(T_{r}-T_{a}\right),
$$

where $V_{r}$ is the AC voltage across the reference sensor with resistance $R_{r}, \alpha_{r}=K g_{r} \mathrm{Nu}_{r}$ is the bulk convective 
heat transfer coefficient, $\mathrm{Nu}_{r}$ is the Nusselt number, $K$ is the thermal conductivity of air, $g_{r}$ is a sensor shapedependent coefficient, $T_{r}$ is the temperature of the reference sensor at the resistance $R_{r}$, and $T_{a}$ is the air temperature.

In cloud, heat losses of the collector sensor result from the wet and dry components:

$$
P_{c}=\alpha_{c}\left(T_{c}-T_{a}\right)+W L * S U,
$$

where $\alpha_{c}=K g_{c} \mathrm{Nu}_{c}$ is the bulk convective heat transfer coefficient for the collector sensor, $T_{c}$ is the temperature of the collector sensor at the resistance $R_{c}, S$ is the collector sample area, $L^{*}$ is the energy expended in heating and evaporating the water, $U$ is the airspeed, and $W$ is the measured water content.

The use of noncoherent currents (i.e., AC and DC) in the collector bridge provides a simple algebraic sum of the powers responsible for dry and wet collector heat losses, so that dry, wet, and total heat losses may be expressed, respectively, as

$$
\begin{aligned}
& P_{c \text { dry }}=\frac{V_{c a}^{2}}{R_{c}}=\alpha_{c}\left(T_{c}-T_{a}\right), \\
& P_{c \text { wet }}=\frac{V_{c d}^{2}}{R_{c}}=W L^{*} S U,
\end{aligned}
$$

and

$$
P_{c \text { tot }}=\frac{V_{c a}^{2}+V_{c d}^{2}}{R_{c}},
$$

where $V_{c a}$ and $V_{c d}$ are AC and DC voltages across the collector sensor with resistance $R_{c}$ and $T_{c}$ is the collector sensor temperature that corresponds to resistance $R_{c}$.

From Eq. (4) we obtain the measured water content:

$$
W=\frac{V_{c d}^{2}}{S U L^{*} R_{c}} .
$$

Note that $W$ found from Eq. (6) must be corrected for collection efficiency $\varepsilon$ and cloud phase.

For liquid cloud, the energy expended in heating and evaporating can be written as

$$
L_{l}^{*}=C_{l}\left(T_{e}-T_{a}\right)+L_{l}\left(T_{e}\right),
$$

where $C_{l}$ is the specific heat of liquid water and $L_{l}\left(T_{e}\right)$ is the latent heat of evaporation of water at the temperature $T_{e}$ of evaporation. In the general case, the temperature of evaporation $T_{e}$ is less than the collector temperature $T_{c}$. The temperature $T_{e}$ can be found from the mass balance equation

$$
U \varepsilon W=\mu\left[E_{s}\left(T_{e}\right)-E_{s}\left(T_{a}\right)\right] .
$$

Here $E_{s}(T)$ is the saturation vapor pressure at temperature $T, \mu$ is the mass transfer coefficient for the collector, and $\varepsilon$ is the sensor collection efficiency. Thus, the evaporation temperature varies in the range $T_{c}<T_{e}$ $<T_{a}$. For simplicity it is convenient to use an average value of $L_{l}^{*}=2580 \mathrm{~J} \mathrm{~g}^{-1}$, which adds a $\pm 5 \%$ error to the LWC for the typical interval of LWC in clouds in the temperature interval from $-40^{\circ}$ to $+20^{\circ} \mathrm{C}$ (Nevzorov 1983).

For ice particles,

$$
L_{i}^{*}=C_{i}\left(T_{0}-T_{a}\right)+L_{i}+C_{l}\left(T_{e}-T_{0}\right)+L_{l}\left(T_{e}\right),(9)
$$

where $C_{i}$ is the specific heat of ice, $L_{i}$ is the latent heat of fusion, and $T_{0}=0^{\circ} \mathrm{C}$. For most applications, the following approximation for Eqs. (7) and (9) can be used: $L_{i}^{*} \approx 1.13 L_{l}^{*}$.

In mixed-phase clouds with the true values of LWC $=W_{l}$ and IWC $=W_{i}$, the measured values of TWC and LWC probes will be, respectively, as follows:

$$
W_{\mathrm{TWC}}=\varepsilon_{l \mathrm{TWC}} W_{l}+1.13 \varepsilon_{i \mathrm{TWC}} W_{i}
$$

and

$$
W_{\mathrm{LWC}}=\varepsilon_{l \mathrm{LWC}} W_{l}+\beta W_{i},
$$

where $\varepsilon_{l \mathrm{TWC}}$ and $\varepsilon_{l \mathrm{LWC}}$ are the liquid droplet collection efficiencies for the TWC and LWC probes, respectively; $\beta \ll 1$ is the factor for the residual effect of the ice on the LWC collector (i.e., the collection efficiency of the liquid sensor for ice particles); and $\varepsilon_{i \mathrm{TWC}}$ is the ice particle collection efficiency for the TWC sensor. Since in most cases IWC is mainly contributed by large particles $(>20 \mu \mathrm{m})$, for which the theoretical TWC collection efficiency should be close to 1 , it can be assumed to a good accuracy $\varepsilon_{i \mathrm{TWC}} \sim 1$. It is logical to speculate that the TWC collection efficiency reduces when the particle size approaches the diameter of the TWC sensor ( $8 \mathrm{~mm})$.

\section{d. Collection efficiency}

\section{1) SMALl LIQUID DROPLETS}

The collection efficiency for the LWC sensor $\varepsilon_{l \mathrm{LWC}}(d)$ for liquid droplets can be theoretically calculated since it has a simple cylindrical shape. Figure 4 shows the collection efficiency calculation based on Voloschuk (1971). For most liquid clouds without large droplets (i.e., droplets $<100 \mu \mathrm{m}$ diameter, much smaller than collector sensor diameter), the integrated collection efficiency for the LWC sensor varies from 0.9 to 1 .

The collection efficiency for the TWC sensor $\varepsilon_{l \mathrm{TWC}}(d)$ for liquid droplets was determined experimentally by Nevzorov (1983) from in situ measurements of LWC as a function of effective droplet diameter $d_{\text {eff }}$. The effective diameter was derived from measurements of the extinction coefficient $G$ and the liquid water content $W_{\text {LWC }}$ measured by the LWC sensor as

$$
d_{\mathrm{eff}}=3 \frac{W_{\mathrm{LWC}}}{\rho_{l} G} .
$$

Here $\rho_{l}$ is the density of liquid water. The extinction coefficient was directly measured by an aircraft cloud transmissometer (Kosarev et al. 1976).

Figure 4 shows this empirically estimated collection efficiency for the TWC sensor for liquid droplets. The 


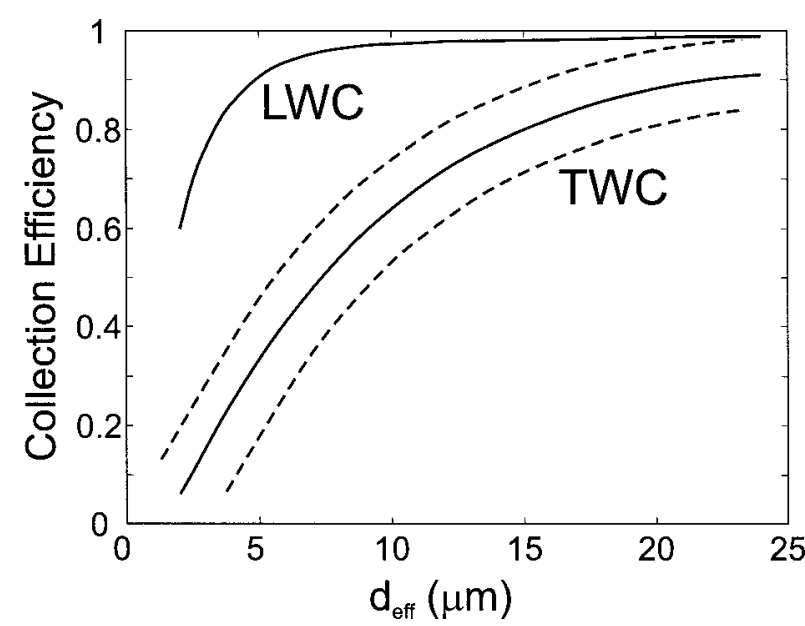

FIG. 4. Dependence of collection efficiency of LWC and TWC sensors for liquid droplets as a function of droplet effective diameter $d_{\text {eff }}$ [Eq. (12)]. Dotted lines are estimated measurement errors of $\pm 15 \%$ for the TWC collection efficiency.

dashed lines show $\pm 15 \%$ error limits due to uncertainties in the measurements. Note that the collection efficiency of the TWC sensor is lower than that of the LWC sensor at all sizes, and the values diverge significantly for small droplets. These results indicate that the uncorrected LWC and TWC values should diverge in clouds with small droplets, which is qualitatively consistent with such observations for the Convair580 probe. However, the magnitude of the difference between the probes' efficiencies in such measurements is smaller than that suggested in Fig. 4, and this issue is currently under reinvestigation. To avoid misinterpretation of collection efficiencies in Fig. 4, note that $d_{\text {eff }} \geq \overline{d_{3}} \geq \bar{d}$. Here $\overline{d_{3}}$ is the mean volume diameter, and $\bar{d}$ is the mean diameter. The three diameters are equal only for a monodisperse droplet size distribution.

The liquid, small-droplet collection efficiency for both sensors can be approximated by the expression (Nevzorov and Shugaev 1992a)

$$
\varepsilon=\frac{d_{\mathrm{eff}}^{2}}{d_{\mathrm{eff}}^{2}+d_{0}^{2}},
$$

where $d_{0}=7.5 \mu \mathrm{m}$ for the TWC collector and $d_{0}=$ $1.7 \mu \mathrm{m}$ for the LWC collector at an airspeed of 100$150 \mathrm{~m} \mathrm{~s}^{-1}$.

\section{2) LARGE LIQUID DROPS}

Biter at al. (1987) have shown that the collection efficiency of the PMS King cylindrical sensing wire approaches unity for droplets greater than $10-\mu \mathrm{m}$ diameter but then decreases as drop sizes reach hundreds of microns, presumably due to the shedding of some of the large-droplet mass after incomplete evaporation of the drop. It is reasonable to assume that the same behavior can be expected for the Nevzorov LWC probe since the diameter of its cylindrical sensor is similar to that of the King probe. The TWC probe, in contrast, is much less likely to shed large droplets since large particles that enter the stagnation region of the TWC conical sensing area have nowhere to go but to the base of the cone. This property of the TWC probe suggests that it may be the only hot-wire sensor currently available that does not seriously underestimate large-droplet mass. Exact characterization of the collection efficiency of the TWC probe for large droplets is yet to be achieved, but a qualitative illustration of the differing response of the LWC and TWC probes in drizzle, consistent with the above argument, is given in section 4 .

\section{3) ICE PARTICLES}

The design of the LWC sensor suggests that ice particles that impact with the sensor should for the most part deflect off the surface with minimal heat transfer. Flight tests in clouds that are arguably composed of only ice particles do show a small LWC signal, presumably resulting from collisions with ice particles. This response is referred to in this article as the "residual signal due to ice" and is discussed further in section $4 \mathrm{~b}(3)$. The collection efficiency of the LWC probe for ice particles in any specific case will depend on the crystal type and size, and it will be very difficult to establish in any general manner due to a lack of calibration standards. Preliminary results indicate that the response of the LWC probe for ice particles is no larger than $15 \%$ of IWC for typical ice phase clouds and for airspeeds of less than $100 \mathrm{~m} \mathrm{~s}^{-1}$ [see section $4 \mathrm{~b}(3)$ ].

The design of the TWC probe, in contrast to the LWC probe, suggests that ice particles that enter the stagnation region of the conical TWC sensing area will be trapped and evaporated. Small ice particles may follow trajectories around the sensor, due to their inability to cross streamlines, and will as a first approximation have a collection efficiency similar to those for small water droplets of equivalent mass (Fig. 4). Once the effective diameters of ice particles are larger than approximately $20 \mu \mathrm{m}$, the collision efficiency of the TWC probe should exceed $80 \%$, which is implied in Fig. 4. Since most of the mass of typical natural ice particle distributions resides at larger sizes, this implies that the TWC sensor should trap most of the ice particle mass. The verification of this near-unity TWC collection efficiency for ice particles is elusive due to the lack of accurate calibration standards and techniques for establishing known natural ice populations. An example illustrating the performance of the TWC probe in a high-speed icing tunnel during the freezing of a spray at a constant water delivery rate is given in section $4 b(1)$. These data seem to indicate a high collection efficiency for small frozen ice spheres, but verification for natural ice populations is as yet unestablished.

In spite of the difficulties in establishing the absolute values of the collection efficiencies of the Nevzorov 
LWC and TWC sensors for liquid and ice, the probe provides unique measurements of the phase separation of mixed clouds in real time and first-order estimates of the phase ratio with uncertainties smaller than those available from other current instrument combinations. Examples of this capability are illustrated in section 4.

\section{Dry air drift tests}

The sensitivity of a hot-wire probe is affected by the accuracy with which the response of the wire to convective heat losses can be removed. An ideal probe will measure exactly $0 \mathrm{~g} \mathrm{~m}^{-3}$ in cloud-free air, but in practice, all hot-wire probes drift from $0 \mathrm{~g} \mathrm{~m}^{-3}$ due to inexact compensation for the convective heat-loss term. This drift is also present in cloud but cannot be separated easily from the liquid water term and therefore affects the accuracy of the LWC calculation. The Nevzorov probe incorporates a separate sensor for the estimate of the convective heat losses in cloud, which is designed specifically to improve compensation and thereby provide a higher sensitivity than conventional hot-wire probes.

The ratio of the powers dissipated by the collector and reference sensors in cloud-free air is

$$
\frac{P_{c \mathrm{dry}}}{P_{r \mathrm{dry}}}=\frac{\alpha_{c}\left(T_{c}-T_{a}\right)}{\alpha_{r}\left(T_{r}-T_{a}\right)} .
$$

Since the reference and collector sensors are maintained at the same temperature (i.e., $T_{r}=T_{c}$ ), this ratio reduces to

$$
\frac{P_{c \mathrm{dry}}}{P_{r \mathrm{dry}}}=\frac{\alpha_{c}}{\alpha_{r}}=k .
$$

Although $\alpha_{c}$ and $\alpha_{r}$ are dependent on temperature, pressure, airspeed, and sensor shape, the dry-air removal scheme makes use of the empirical result that their ratio $k$ is approximately constant. Removal of the dry-air convective heat loss (balancing) of the Nevzorov probe is accomplished by adjusting the coefficient $k$ using the potentiometer 6 (Fig. 3) in cloud-free air so that

$$
k P_{r \text { dry }}-P_{c \text { dry }}=0 .
$$

In reality, the dependent parameters affect the coefficient $k$ to some degree. The resulting baseline LWC and/ or TWC drift affects the zero reading out of cloud and the accuracy and sensitivity of the measurements in cloud. Although baseline drift appears to be minimized in the Nevzorov probe, the design of the electronics necessitates that the probe be run with a positive offset in order to monitor drift, and drifts toward an excess in $P_{r \text { dry }}$ (negative drift) are recorded as a zero drift and are currently unrecoverable. Therefore, to obtain the most accurate results, it is necessary for an operator to monitor the instrument and make periodic baseline adjustments.

The magnitude of baseline drift effects is examined
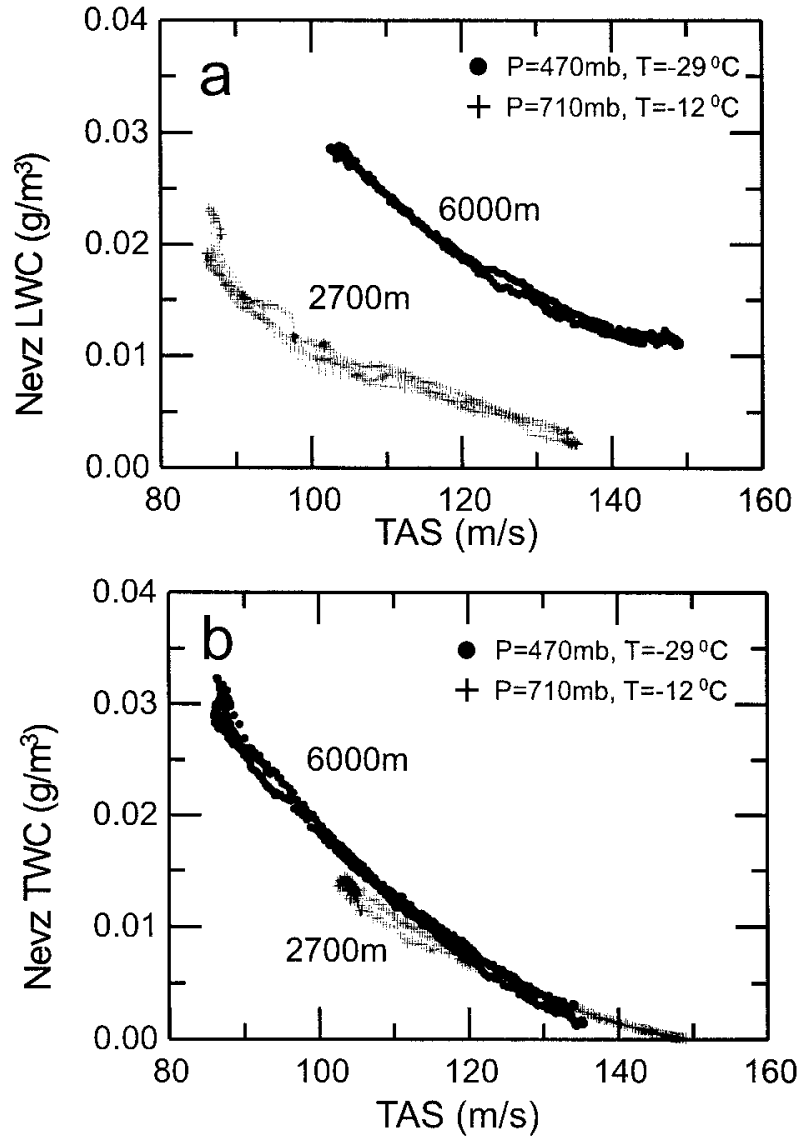

FIG. 5. Dry-air baseline drift as a function of airspeed at 2700 and $6000 \mathrm{~m}$ for the (a) LWC and (b) TWC sensors, expressed as an error in grams per cubic meter.

in the following sections. The baseline drift may vary slightly from sensor to sensor. It also depends on the aerodynamics of a particular location of a sensor head on the aircraft since changes of airspeed at the location of the sensor head may be not linearly related to changes of airspeed of the aircraft. On the NRC Convair580, the Nevzorov probe was mounted on a pylon $0.5 \mathrm{~m}$ below the wing and ahead of its leading edge.

\section{a. Drift with airspeed}

Figures $5 \mathrm{a}$ and $5 \mathrm{~b}$ illustrate the baseline drift of the LWC and TWC sensors, expressed as water content, due to airspeed changes in cloud-free air. The measurements were collected by the NRC Convair580 aircraft for two altitudes of 2700 and $6000 \mathrm{~m}$. The slope of curves appear to be independent of altitude in this range and are approximately $\partial W / \partial u \sim-2 \times 10^{-3} \mathrm{~g}$ $\mathrm{m}^{-3} / 10 \mathrm{~m} \mathrm{~s}^{-1}$ for the LWC sensor and $\partial W / \partial u \sim-3 \times$ $10^{-3} \mathrm{~g} \mathrm{~m}^{-3} / 10 \mathrm{~m} \mathrm{~s}^{-1}$ for the TWC sensor. For the normal $20 \mathrm{~ms}^{-1}$ true airspeed range of the Convair580 aircraft during cloud physics studies, this corresponds to a drift of the order of 0.004 and $0.006 \mathrm{~g} \mathrm{~m}^{-3}$ for the LWC and TWC probes, respectively. 


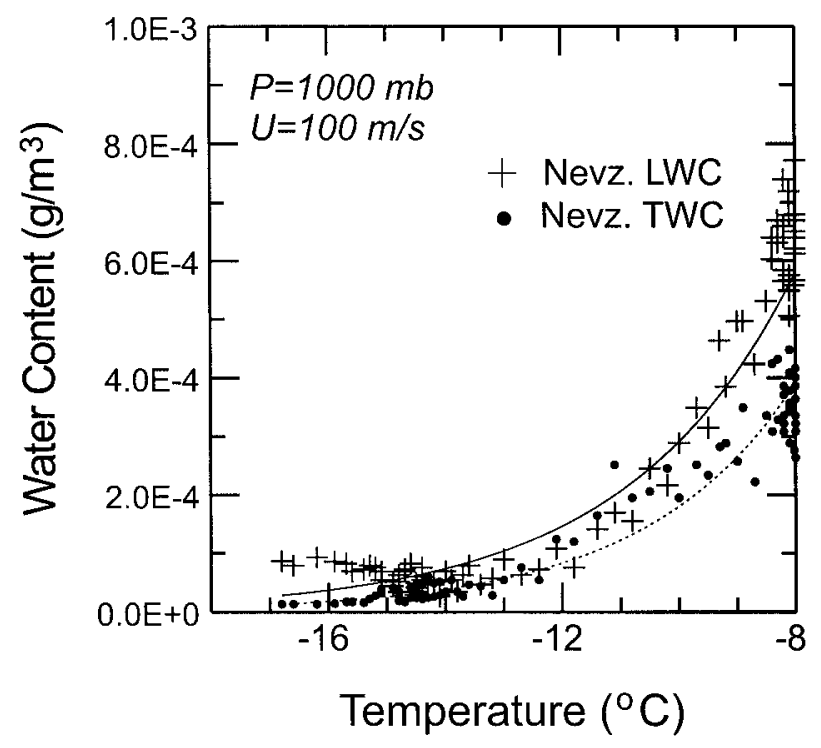

FIG. 6. Dry-air baseline drift as a function of temperature, expressed as an error in grams per cubic meter. Measurements were collected at the NRC wind tunnel: $P=1000 \mathrm{mb}$ and $U=100 \mathrm{~m} \mathrm{~s}^{-1}$.

\section{b. Drift with air temperature}

The baseline drift due to temperature changes was evaluated in wind tunnel tests performed at the NRC high-speed icing wind tunnel (Fig. 6). At an airspeed of $100 \mathrm{~m} \mathrm{~s}^{-1}$ and $p=1000 \mathrm{mb}$, a linear estimate of the variation of the baseline drift was estimated to be $\partial W /$ $\partial T \sim 0.5 \times 10^{-3} \mathrm{~g} \mathrm{~m}^{-3}\left(10^{\circ} \mathrm{C}\right)^{-1}$ for both the LWC and TWC probes, in the temperature interval from $-20^{\circ}$ to $-5^{\circ} \mathrm{C}$. Temperature effects therefore appear to be of secondary importance and insignificant for all levels of flight conditions.

\section{c. In-flight altitude-temperature drift}

Figure 7 shows variations of measured water content during a constant true airspeed ascent of the Convair580 from 3 to $6 \mathrm{~km}$. In this cloud-free region the temperature changed from $-12^{\circ}$ to $-29^{\circ} \mathrm{C}$ during the ascent. The baseline drift on both the TWC and LWC probes displayed the same dependence on altitude-temperature of $\partial W / \partial Z \sim 5 \times 10^{-3} \mathrm{~g} \mathrm{~m}^{-3} \mathrm{~km}^{-1}$. Since the temperature effect on the baseline drift $(\partial W / \partial T) \Delta T$ is an order of magnitude less (section $3 b)$ than the value $(\partial W / \partial Z) \Delta Z$, it follows that $\partial W / \partial Z$ depends mainly on pressure. For a typical $3-\mathrm{km}$ aircraft vertical sounding, the baseline drift for both probes can be expected to reach $0.015 \mathrm{~g}$ $\mathrm{m}^{-3}$.

\section{d. Random clear-air fluctuations}

Baseline drift in cloud-free sky during normal-level horizontal flight at constant airspeed was measured on the Convair580 under relatively low turbulence levels. During these tests, both the LWC and TWC probes were

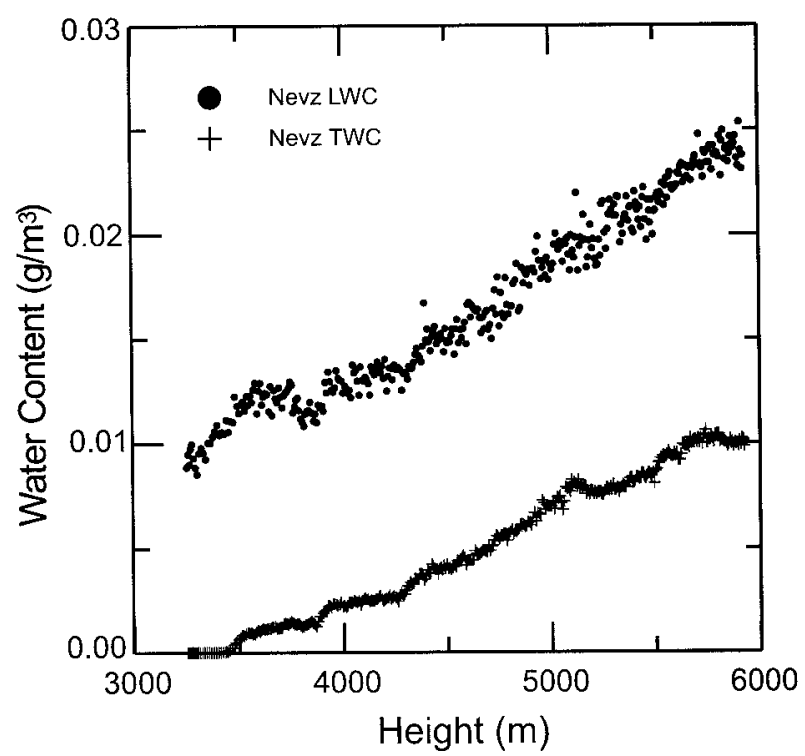

FIG. 7. Dry-air baseline drift as a function of altitude expressed as an error in grams per cubic meter; $U=105 \mathrm{~m} \mathrm{~s}^{-1}$.

deliberately operated with a small, positive offset in order to observe fluctuations around an average positive value. Figure 8 contains a 25 -min time record of these data, along with a histogram of the 1-s interval, clearair LWC and TWC values. Maximum deviations from mean value are also shown as $\pm 0.002 \mathrm{~g} \mathrm{~m}^{-3}$ for both probes, which define the threshold sensitivity of the probe. It is expected that these deviations will increase with increasing turbulence. This drift normally appears as excursions with periods of several seconds, and the normal 1-s random fluctuations are much lower than $0.001 \mathrm{~g} \mathrm{~m}^{-3}$.

The measurements of the dry-heat term in clear air conducted by King et al. (1981) showed that the maximum value of the random fluctuations of the CSIRO PMS King probe was $\pm 0.02 \mathrm{~g} \mathrm{~m}^{-3}$ in terms of equivalent LWC. This is an order of magnitude higher than that for the Nevzorov probe.

\section{Illustrations of probe performance in cloud}

The data shown in the subsequent sections are intended to provide typical examples of the performance of the Nevzorov probe and cursory comparisons to other instruments in order to illustrate basic reliability and unique capabilities of the instrument. However, they are not intended as indications of the absolute accuracy of the measurement. Collection efficiencies in liquid cloud have not been applied to either the Nevzorov or the King probe data. The LWC sensor wires of both probes are approximately the same diameter, and the TWC collection efficiency is currently under reinvestigation. The collection efficiency for ice particles for the LWC and TWC sensors have been assumed to be 0 and 1, respectively. In mixed-phase clouds, calculations of TWC 

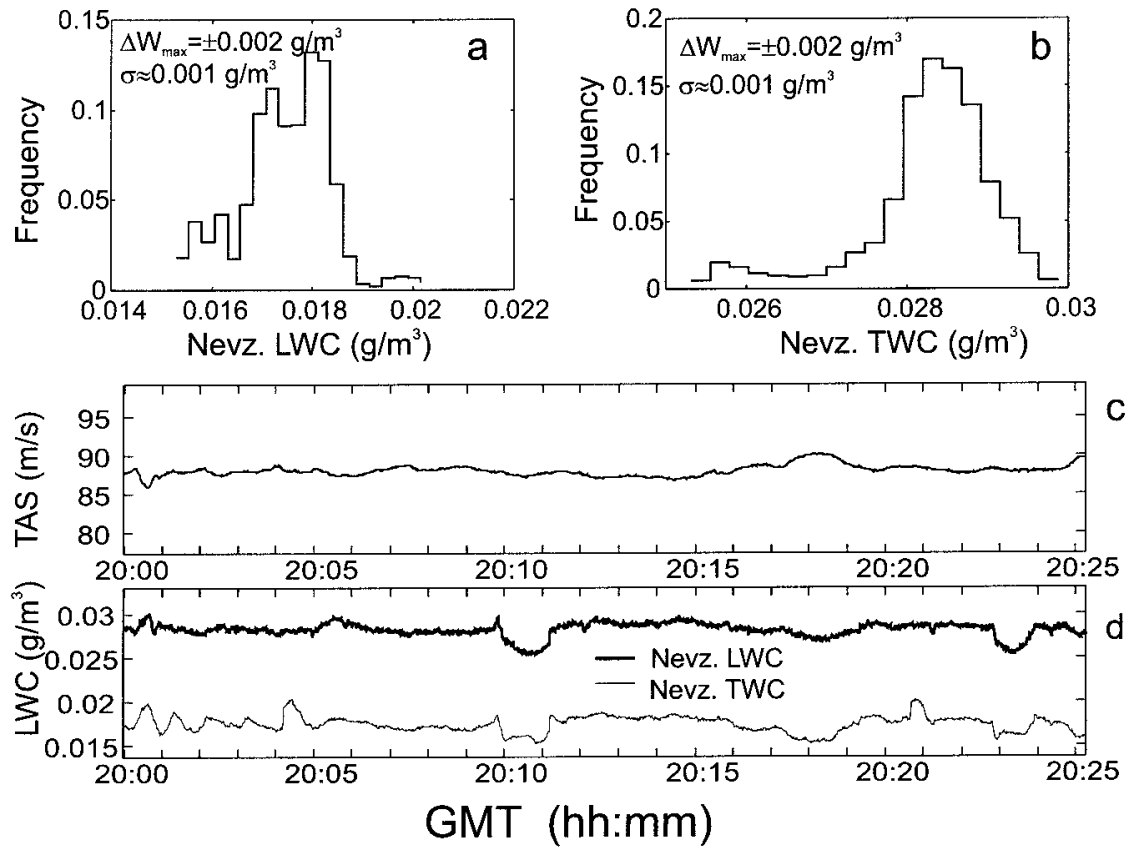

FIG. 8. Frequency distribution of the $1-\mathrm{Hz}$ (a) LWC and (b) TWC values of (d), illustrating the random drift in straight and level dry-air flight: $H=2700$ and $T=-12^{\circ} \mathrm{C}$. (c) Time variations of true airspeed and (d) time histories of the $1-\mathrm{Hz}$ LWC and TWC signals.

have been performed assuming a liquid cloud, and no attempt has been made to make adjustments for mixedphase heat transfer condition, as suggested in Eqs. (10) and (11). For example, disregarding corrections due to the latent heat of fusion and the specific heat of ice in Eq. (9) causes errors in the TWC of about $13 \%$ due to the latent heat of fusion alone.

\section{a. Liquid clouds}

Intercomparisons between the Nevzorov probe, the King probe, and the PMS Forward Scattering Spectrometer Probe (FSSP) have been performed in liquid clouds during several projects with the Convair580. Figure 9 shows typical comparisons of the Nevzorov and
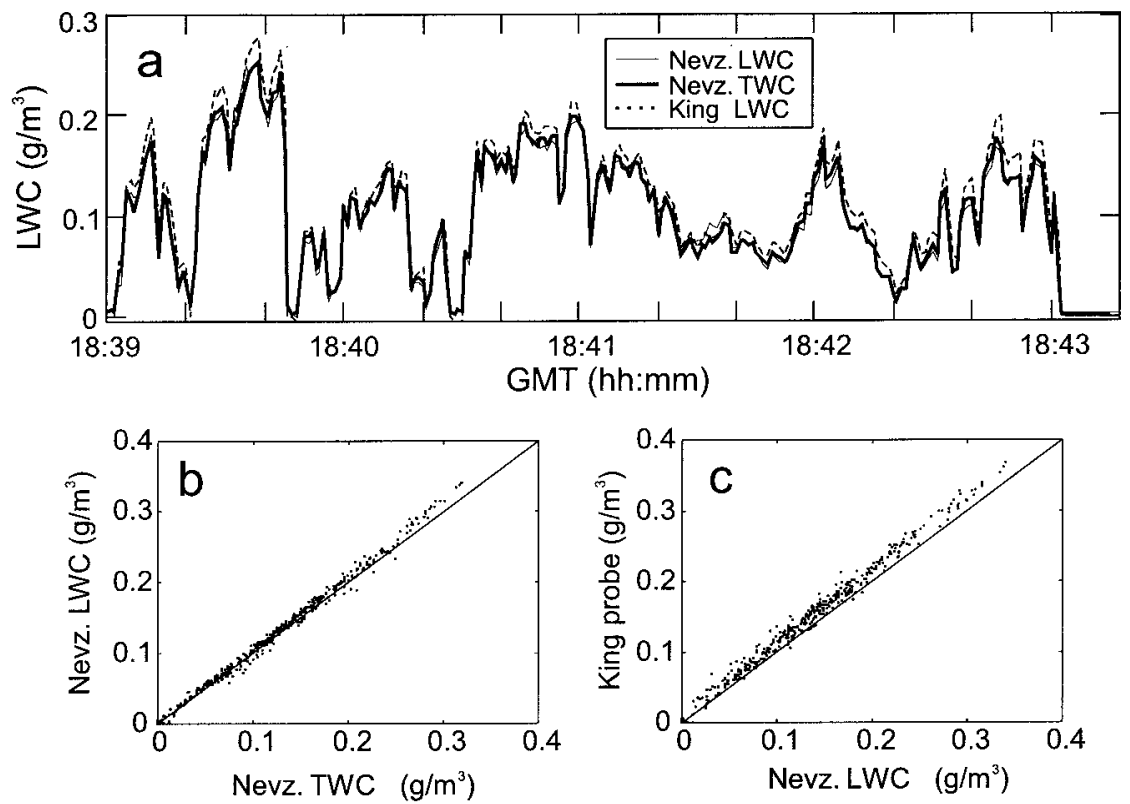

FIG. 9. Comparisons of Nevzorov LWC-TWC and King probes in liquid stratus clouds: $H=$ $800 \mathrm{~m} ; T=-5^{\circ} \mathrm{C} ; 17$ March 1995 , Newfoundland. 

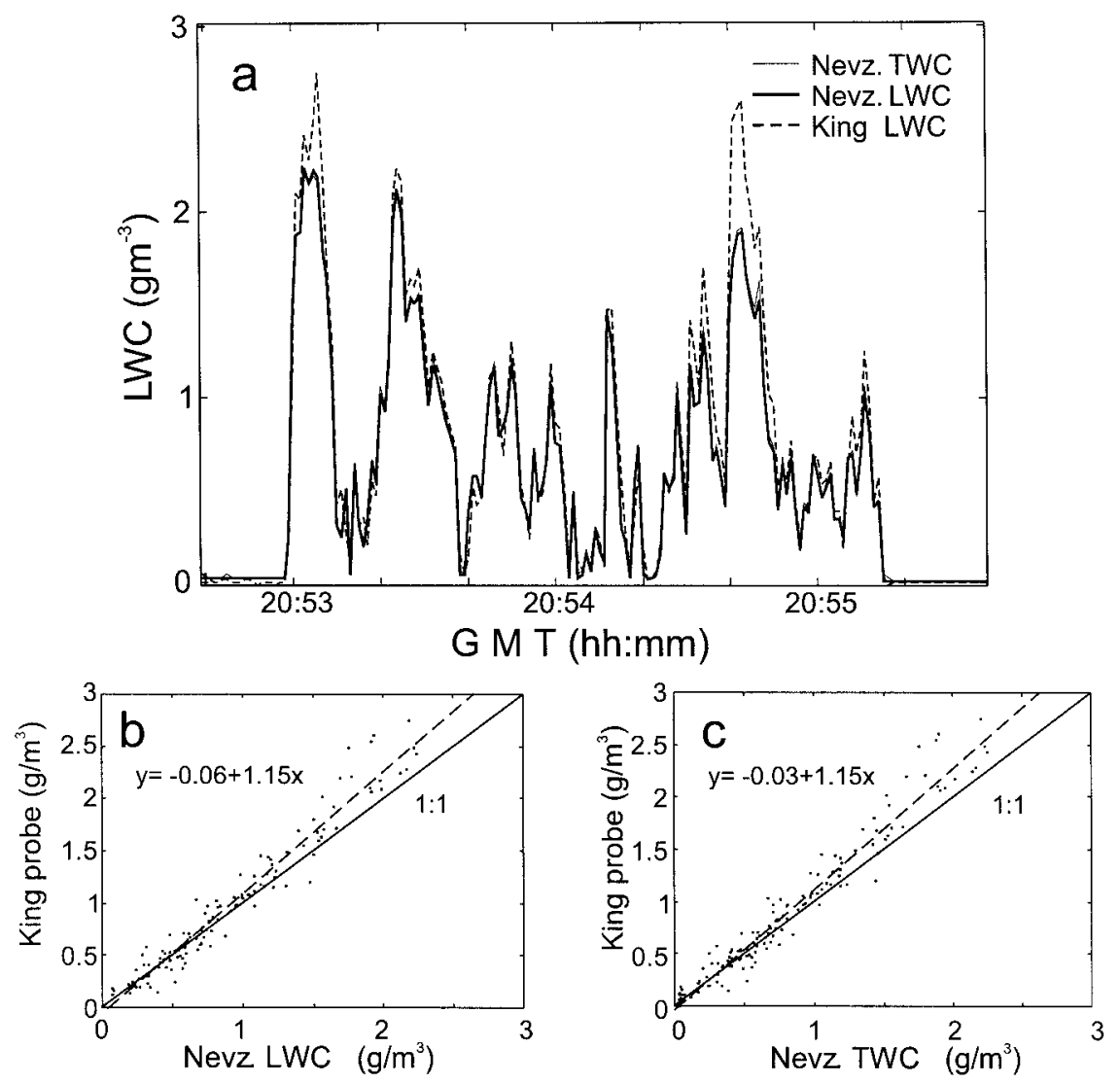

FIG. 10. Comparisons of Nevzorov LWC-TWC and King probes in liquid cumulus congestus clouds: $H=3000 \mathrm{~m} ; T=+4^{\circ} \mathrm{C} ; 18$ August 1994, Ottawa.

King probe measurements in low-LWC stratiform clouds during the Canadian Freezing Drizzle Experiment. The scatterplots shown in Figs. 9b and 9c demonstrate good agreement between the Nevzorov and King probes. The slope of the regression line forced through the origin for the Nevzorov LWC and TWC comparison is 1.04 , reflecting the basic uncertainties in the calculations and perhaps the uncertainty in collection efficiency. The regression line for the Nevzorov LWCPMS King probe comparison reveals a slight gain difference but also an indication of a slight positive offset. This kind of offset was frequently observed in Nevzorov-King comparisons and is attributed to the superior baseline discrimination for the Nevzorov probe, especially in cases of long traverses through cloud with few cloud-free pockets for baseline reference. LWC estimates from all three probes tend to vary by less than $15 \%$.

Figure 10 demonstrates the performance of the Nevzorov probe in high liquid water content clouds. The measurements were performed in liquid cumulus congestus clouds at a temperature $T=+4^{\circ} \mathrm{C}$ near Ottawa, Canada. Figure 10 shows a good agreement between Nevzorov LWC-TWC and King probes at LWC up to
$2 \mathrm{~g} \mathrm{~m}^{-3}$. The scatterplots of Figs. 10b and 10c reveal what is most easily described as a scale factor difference between the King and Nevzorov probes with no convincing evidence of a nonlinear trend. Both the Nevzorov LWC and TWC probes read lower than the King probe by approximately $15 \%$. All three measurements are made within $30 \mathrm{~cm}$ of each other on the Convair 580 , so that it is unlikely that the LWC difference is due to mounting location. It does, however, appear likely that uncertainty in the basic calibration parameters of the sensors (e.g., sensor sample area) contributed to the scale factor difference.

It is interesting to note that the PMS King probe exceeds the Nevzorov LWC and/or TWC in both Figs. 9 and 10, and it is also overall more common to observe this tendency in other liquid-cloud comparisons. Such differences may also be partially explained by wetting of the Nevzorov reference sensors, especially in high LWC clouds, or some other unknown factor related to either probe. Although it is contended that the difference between the King and Nevzorov probes in liquid cloud should be less than $15 \%$ for sensors with well-measured basic calibration parameters, further research would be 


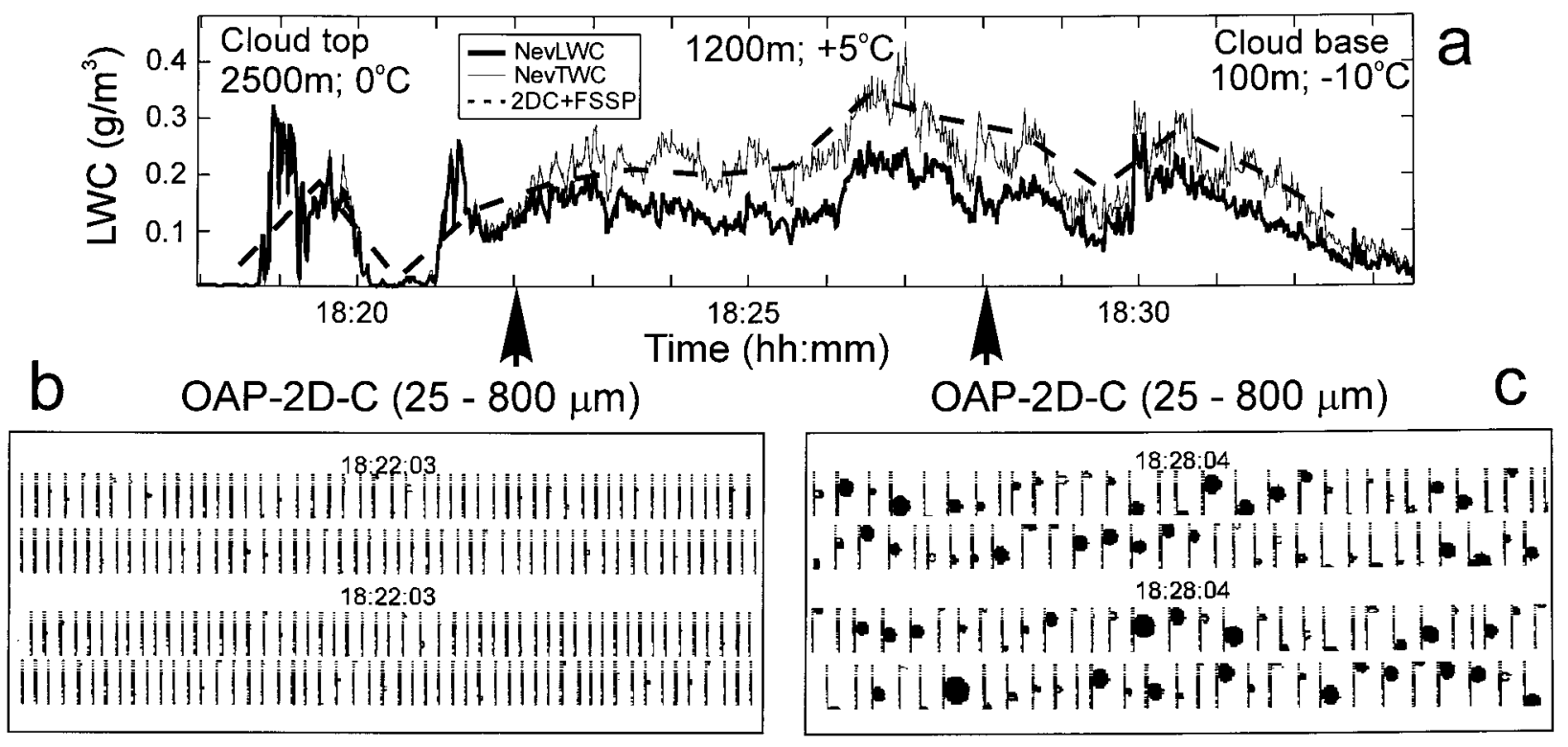

FIG. 11. (a) Variations of Nevzorov LWC, TWC, and 1-min-averaged LWC derived from FSSP and OAP-2D-C during descent through a drizzling nimbostratus cloud in Newfoundland on 9 March 1995, illustrating the superior response of the TWC probe to drizzle. Right and left arrows near the $x$ axis correspond to 2D images of drops shown in (b) and (c), respectively. A vertical bar of length $800 \mu \mathrm{m}$ precedes each image in (b) and (c): cloud top $H=2500 \mathrm{~m}$ and $T=-2^{\circ} \mathrm{C}$, cloud base $H=100 \mathrm{~m}$ and $T=-10^{\circ} \mathrm{C}$, respectively.

required to isolate the source of these remaining differences.

Figure 11a displays data from a descent by the Convair580 into nimbostratus cloud. Using PMS OAP-2D$\mathrm{C}$ data, it was determined that this cloud layer contained mostly small droplets at cloud top (Fig. 11b) and heavy drizzle lower in the cloud (Fig. 11c). The sample PMS 2D-C imagery displays drizzle drops up to approximately $600 \mu \mathrm{m}$ in the middle of the cloud (Fig. 11c). Note that the TWC is approximately equal to the LWC at cloud top but is almost a factor of 2 higher in the area of heavy drizzle. Biter et al. (1987) have shown that the PMS King probe has a reduced efficiency for droplets in the drizzle size range. Since the Nevzorov LWC probe is also cylindrical, with only a slightly smaller diameter than that of the PMS King probe, it is reasonable to assume that the Nevzorov LWC probe will also underestimate LWC when it is concentrated in large drops. The geometry of the Nevzorov TWC sensor, in contrast, suggests that it will trap the total mass of any large droplets that enter the stagnation region of its conical sample area and should therefore exhibit a larger collection efficiency than that of the LWC sensor. Oneminute-averaged LWC combined from FSSP and OAP2D-C (dashed line in Fig. 11a) supports the Nevzorov TWC measurements. This case illustrates the unique capability of the TWC probe to make LWC measurements beyond the range of conventional hot-wire devices due to its aerodynamic properties.

An agreement of 5\%-20\% was also found in most cases of low LWC measured by Nevzorov and that de- rived from FSSP data. However, in some cases the ratio between the Nevzorov LWC probe and FSSP were found to be greater than a factor of 2. This occurred when droplets contributing a major fraction of the LWC are out of the FSSP size range. Figure 12 shows an intercomparison between the FSSP, King, and Nevzorov probes obtained during vertical soundings in a marine stratus layer. The agreement between the King and Nevzorov probes is within $10 \%$ for the whole leg shown in Fig. 12b. The agreement between the FSSP and Nevzorov probe does not exceed $10 \%$ in the left section of Fig. 12c, where the whole droplet size spectrum is within the FSSP size range $(2-32 \mu \mathrm{m})$ (Fig. 12d). However, in the right section of Fig. 12c the ratio of the Nevzorov LWC and FSSP in some places (e.g., at 1824 and 1827 GMT) can be as high as factor of 10. Figure 12e indicates that in this part of the cloud the major fraction of droplet size distribution is outside of the FSSP size range. Some differences between the Nevzorov LWC and TWC probe readings in the right section is caused by the presence of drizzle.

\section{b. IWC measurements}

\section{1) WIND TUNNEL TESTS}

The response of the LWC and TWC sensors to ice particles was tested at the NRC (Ottawa) high-speed icing wind tunnel. The median volume diameter during this test was approximately $20 \mu \mathrm{m}$. While maintaining a relatively constant water volume flow rate and, pre- 

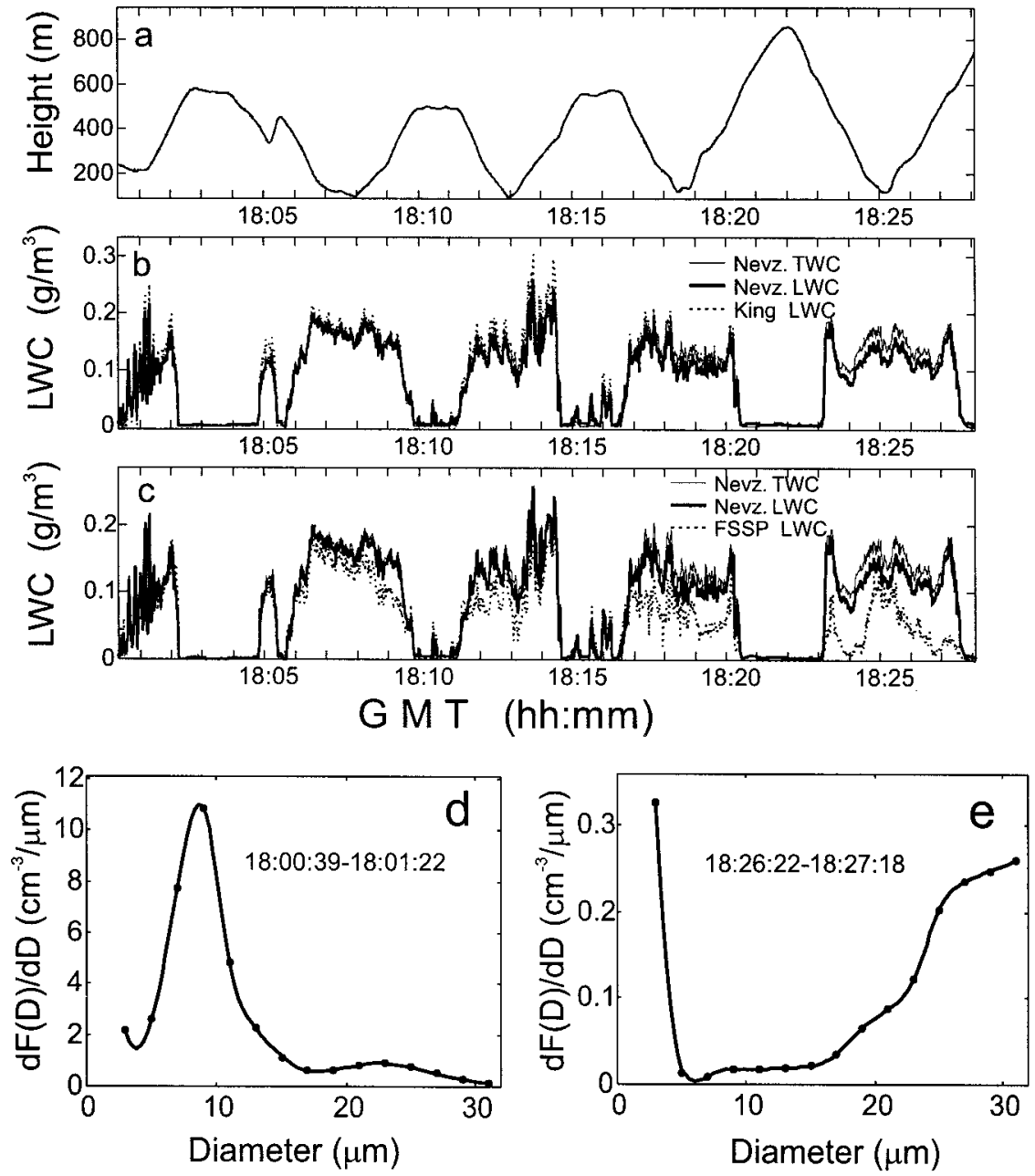

FIG. 12. Time histories of (a) altitude; (b) Nevzorov, LWC-TWC, and King probes water contents; and (c) Nevzorov, LWC-TWC, and LWC derived from FSSP-size range 2-32 $\mu \mathrm{m}$. (d) and (e) Averaged FSSP droplet size distributions corresponding to the left and right sections of (c) with small and large droplets, respectively. Newfoundland, St, $T=-2^{\circ}-0^{\circ} \mathrm{C}, 2$ March 1995.

sumably, a constant spatial distribution in the tunnel cross section, the temperature in the wind tunnel was decreased, eventually producing a partial glaciation of the spray. A time history of the Nevzorov and King probe response and the trace of tunnel static temperature are shown in Figs. 13a and 13b, respectively. The TWC stayed approximately constant, while the LWC measured by the Nevzorov and King probes decreased rapidly as the temperature dropped below $-2^{\circ} \mathrm{C}$. At the outset, the King probe signal was higher than the Nevzorov LWC and TWC because in this case it was situated in a different region of the spray plume. The residual signal of the Nevzorov LWC and PMS King probes at cold temperatures may be explained by the incomplete freezing of the spray. The presence of unfrozen droplets was confirmed by the observation of ice buildup on undeiced areas of the probes. This test clearly verifies that the aerodynamic properties of the Nevzorov LWC and TWC sensors allow for phase separation, at least under the specific condition of a cloud composed of small ice spheres to an accuracy of approximately $10 \%-$ $20 \%$. Verification in natural snow and ice particle populations currently cannot be obtained due to the lack of any accurate reference instrument or standard calibration source for these types of hydrometeors.

\section{2) Mixed-phase cloud Measurements}

The Nevzorov probe exhibits diverging and converging LWC and TWC response in natural clouds that is consistent with separation of ice and liquid zones in mixed clouds. The example presented in Fig. 14 illustrates a flight in mixed nimbostratus clouds. On the left and right sections of the graph, cells of nearly glaciated cloud are observed (TWC $\gg$ LWC). LWC response in these regions may be due to the residual effect of ice 

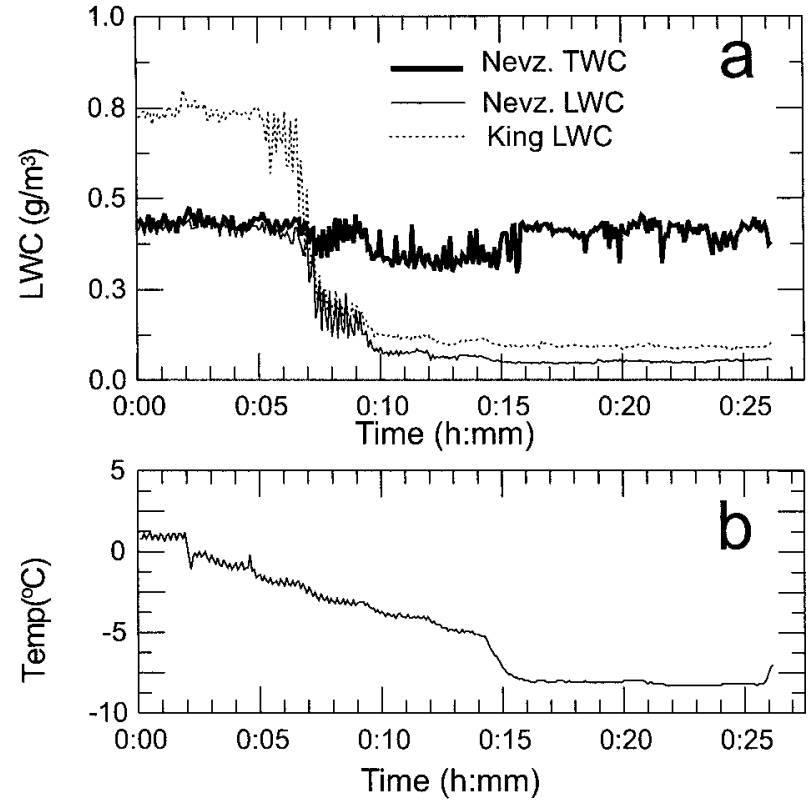

FIG. 13. Glaciation of a constant water spray condition at the NRC high-speed icing tunnel in Ottawa. (a) Variation of Nevzorov, LWCTWC, and PMS King sensor measurements during the phase change from liquid to ice particle conditions and (b) decrease in tunnel static temperature during the test.

particles on the LWC sensor [section $4 b(3)$ ]. The sample PMS OAP-2D-C imagery displays an abundance of ice particles in these zones (Figs. 14c and 14d). Near 1543 and 1545 GMT there are two cells of nearly all liquid cloud, where LWC and TWC measurements are nearly equal. The phase conditions of these zones derived from Nevzorov measurements are consistent with those indicated by the measurements of the Rosemount ice detector (RICE) in Fig. 14b. The ice detector measures the vibration frequency of an exposed rod, which changes when ice is accreted and results in an increase output signal. Once sufficient ice has built up, the rod deices (e.g., Fig. 14b, 1545 GMT) and the cycle starts again. In Fig. 14, when the Nevzorov probe indicates an ice cloud, the RICE signal is constant or decreases. When the Nevzorov probe indicates a cloud with significant LWC, the RICE signal increases.

An additional example of measurements in mixed clouds is shown in Fig. 15a. The measurements were collected by the Convair580 in the upper part of a cumulus congestus. The IWC for this case in some places reaches $1.5 \mathrm{~g} \mathrm{~m}^{-3}$. The LWC occasionally exceeds 0.3 $\mathrm{g} \mathrm{m}^{-3}$, although a fraction of this response may result from the residual effect of the high ice mass concentration on the LWC sensor [section 4b(3)]. Figure $15 \mathrm{~b}$ illustrates OAP-2D-C images of ice particles in this cloud. It is interesting to note that the ratio of LWCTWC was approximately constant in cloud and increased toward the cloud edges (Fig. 15c). This kind of observation may prove useful in understanding the factors affecting ice formation and distribution in cloud.

\section{3) RESiduAl EFFECT OF THE ICE ON THE LWC SENSOR}

The residual effect of ice on the LWC sensor results from the small amount of heat removed from the LWC sensor during collision with ice particles. One could speculate that these collisions consist of momentary interactions as a particle strikes and bounces off the wire but could also include less transient interactions when ice particles strike the stagnation point of the wire. Figure 16 shows measurements of the Nevzorov probe and the RICE in a glaciated cloud. Small variations of the RICE signal indicate that LWC in the cloud does not exceed $0.01 \mathrm{~g} \mathrm{~m}^{-3}$ (Heymsfield 1989). The Nevzorov LWC in this cloud varies from 0.001 to $0.025 \mathrm{~g} \mathrm{~m}^{-3}$ and exceeds the sensitivity threshold of the RICE. This implies that the LWC signal is due to response to ice crystals or liquid droplets with LWC less than $0.01 \mathrm{~g}$ $\mathrm{m}^{-3}$, and the magnitude of this residual ice effect can be estimated by comparing the LWC response to the TWC measurements (Fig. 16d). The linear regression forced through the origin yields a relationship of LWC $=0.06$ TWC. For other clouds, the corresponding regression slope varies between 0.05 and 0.15 , and, on the average, the residual effect of ice is of the order of $10 \%$ of the IWC. Nevzorov and Shugaev (1992b) found a weaker effect, not exceeding $2 \%-3 \%$ of the TWC in their observations of cold clouds. The effect may be expected to vary due to differences in particle habits and bulk density, and intuitively could be expected to be stronger at higher airspeeds $\left(>100 \mathrm{~m} \mathrm{~s}^{-1}\right)$. The response of the Nevzorov LWC probe to ice is similar to that observed for the PMS King probe by Cober et al. (1998, manuscript submitted to J. Atmos. Oceanic Technol.), who found that that the latter probe exhibited a response of approximately $20 \%$ of the Nevzorov TWC in glaciated cloud.

The above analysis of the residual effect of ice assumes that the clouds were totally glaciated. However, the conventional measurements used to identify glaciated cloud cannot detect trace amounts of LWC less than $0.01 \mathrm{~g} \mathrm{~m}^{-3}$ in mixed clouds. Therefore, it is possible that some true LWC response is superimposed on the residual estimates above, exaggerating the problem to a degree. Nevertheless, due to the potential importance of even small amounts of LWC to the physical interpretation of otherwise glaciated clouds, further investigation is warranted to more fully characterize this effect.

\section{Conclusions}

The Nevzorov LWC-TWC is a hot-wire device whose fundamental response, like that of the PMS King probe, is calculable from simple first principles of convective 


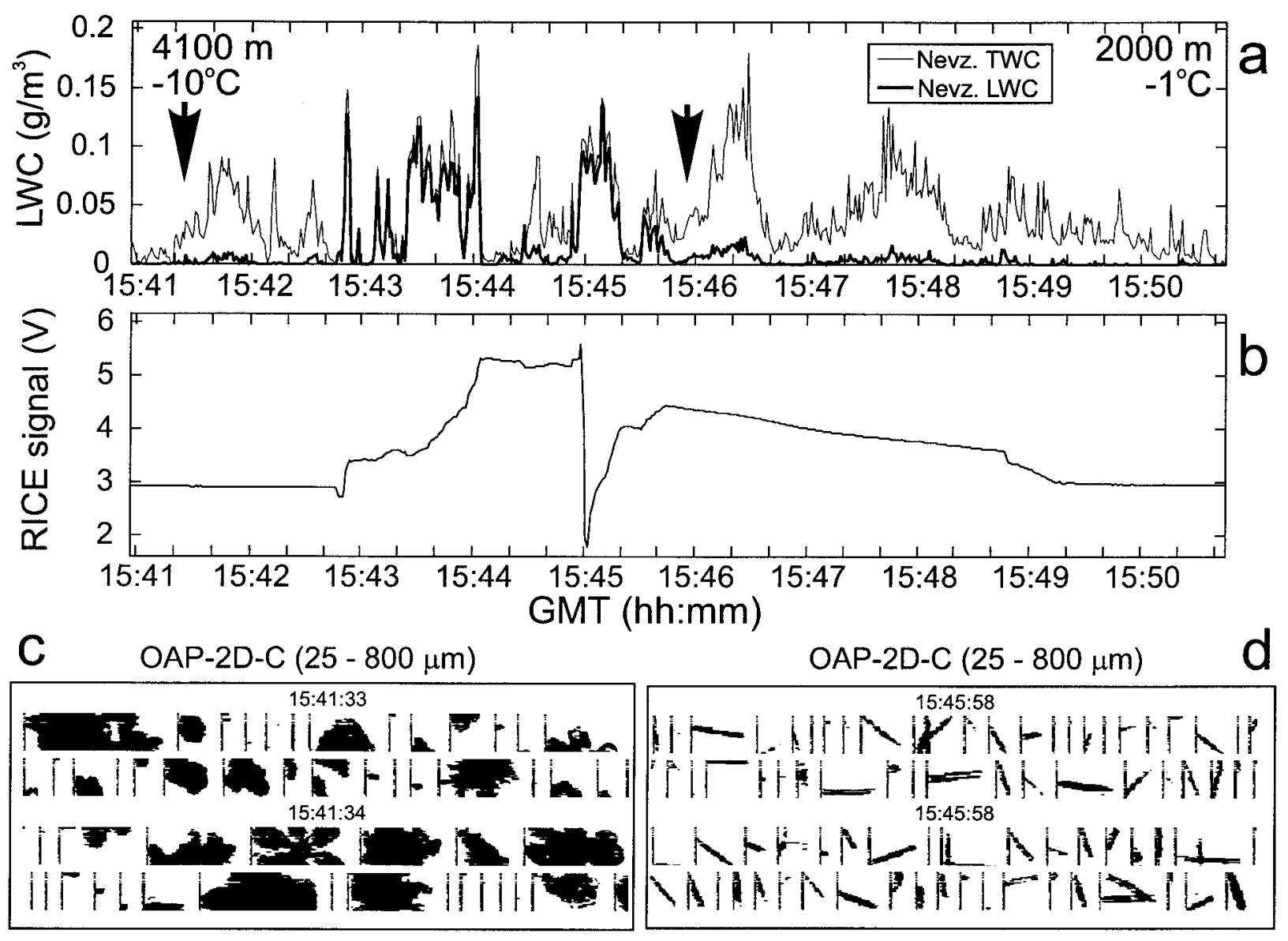

FIG. 14. Time history of the Nevzorov, LWC, and TWC probes in mixed nimbostratus: $H=3500 \mathrm{~m}, T=-5^{\circ} \mathrm{C}, 10 \mathrm{March} 1995$, Newfoundland. Two liquid water regions are clearly seen in the central part of the figure at 1543:30 and 1545:00 GMT. Two-dimensional images in (c) and (d) are for the times of the left and right arrows, respectively, in (a).

heat exchange and evaporation of impacting particles. The specific aerodynamic designs of the separate LWC and TWC sensors of the probe result in its unique capability to separate ice and liquid regions in mixedphase clouds. Such information can be easily provided in real time during flight missions. The Atmospheric Environment Service has collected a large dataset with the probe during several research experiments conducted with the NRC Convair580 aircraft and at the NRC high-speed icing wind tunnel since 1993. Errors in water content due to baseline drift attributed to variations of temperature, pressure, and airspeed are of the order of $\partial W / \partial T \sim 0.5 \times 10^{-3} \mathrm{~g} \mathrm{~m}^{-3}\left(10^{\circ} \mathrm{C}\right)^{-1}, \partial W / \partial Z$ $\sim 5 \times 10^{-3} \mathrm{~g} \mathrm{~m}^{-3} \mathrm{~km}^{-1}$, and $\partial W / \partial u \sim 3 \times 10^{-3} \mathrm{~g} \mathrm{~m}^{-3}$ $\left(10 \mathrm{~m} \mathrm{~s}^{-1}\right)^{-1}$, respectively. The full-flight unattended drift of the probe is estimated at $0.035 \mathrm{~g} \mathrm{~m}^{-3}$ for the typical Convair580 operating range. The random noise of the Nevzorov baseline in straight and level flight, however, is estimated at $\pm 0.002 \mathrm{~g} \mathrm{~m}^{-3}$, which is at least a factor of 5 less than similar measurements for the Atmospheric Environment Service King probe, illus- trating the advantage of the sensitive, dry-air compensation by the reference wire.

The measurements of LWC in liquid clouds by hotwire probes depend on the shape of the collector sensor, droplet size distribution, airspeed, air pressure, and temperature. These parameters define the collection efficiency and thus affect the errors in measurements of LWC. Therefore, the accuracy of a hot-wire probe depends on the cloud microstructure. Even the same droplet size distribution sampled at different altitudes and airspeeds may result in different LWCs measured by the same probe. Thus, any conclusion on the accuracy of LWC measurement without citing the droplet size range, the airspeed, and the pressure is not particularly meaningful. We estimate an absolute accuracy of the Nevzorov LWC-TWC probe as $10 \%-15 \%$ for liquid droplets in the size interval 10-50 $\mu \mathrm{m}$ (where collection efficiency is close to 1) at the airspeed $100 \mathrm{~m} \mathrm{~s}^{-1}$ and pressure $1000 \mathrm{mb}$.

Intercomparisons of the Nevzorov LWC, TWC, and PMS King probes display good agreement in nonprecipitating liquid clouds. The difference between the Nev- 


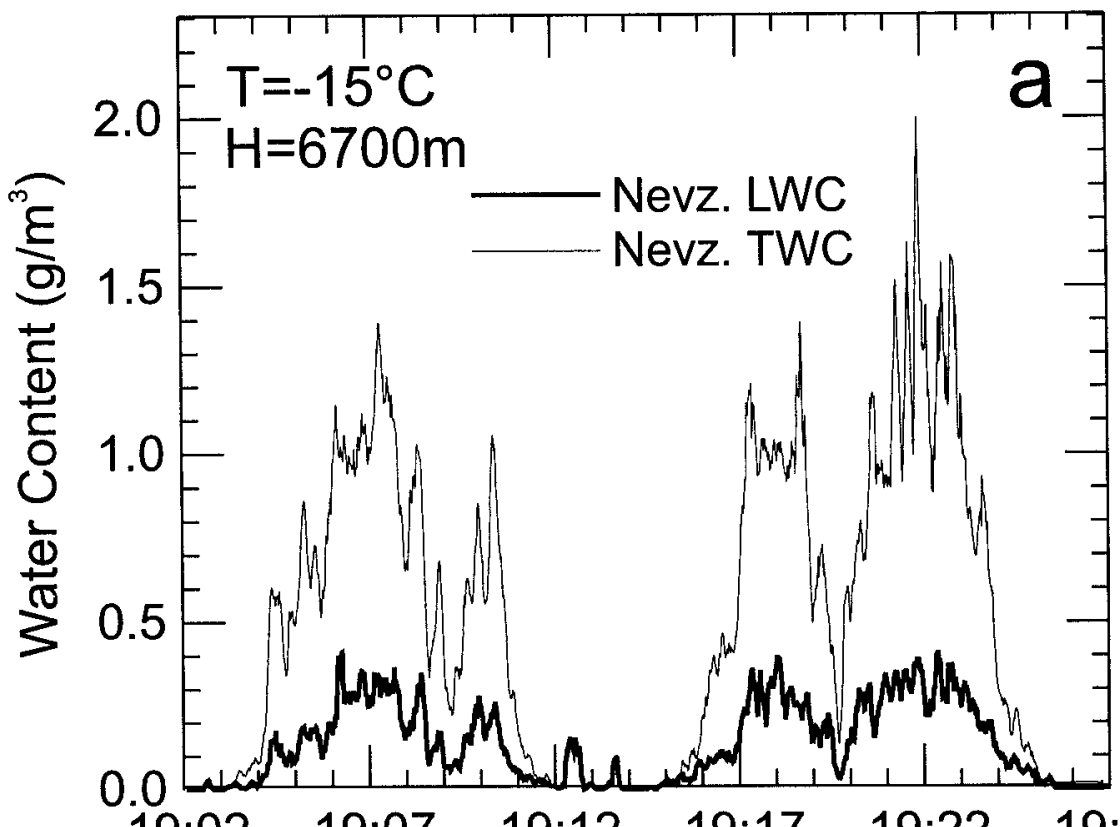

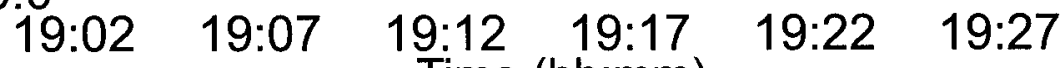

Time (hh:mm)
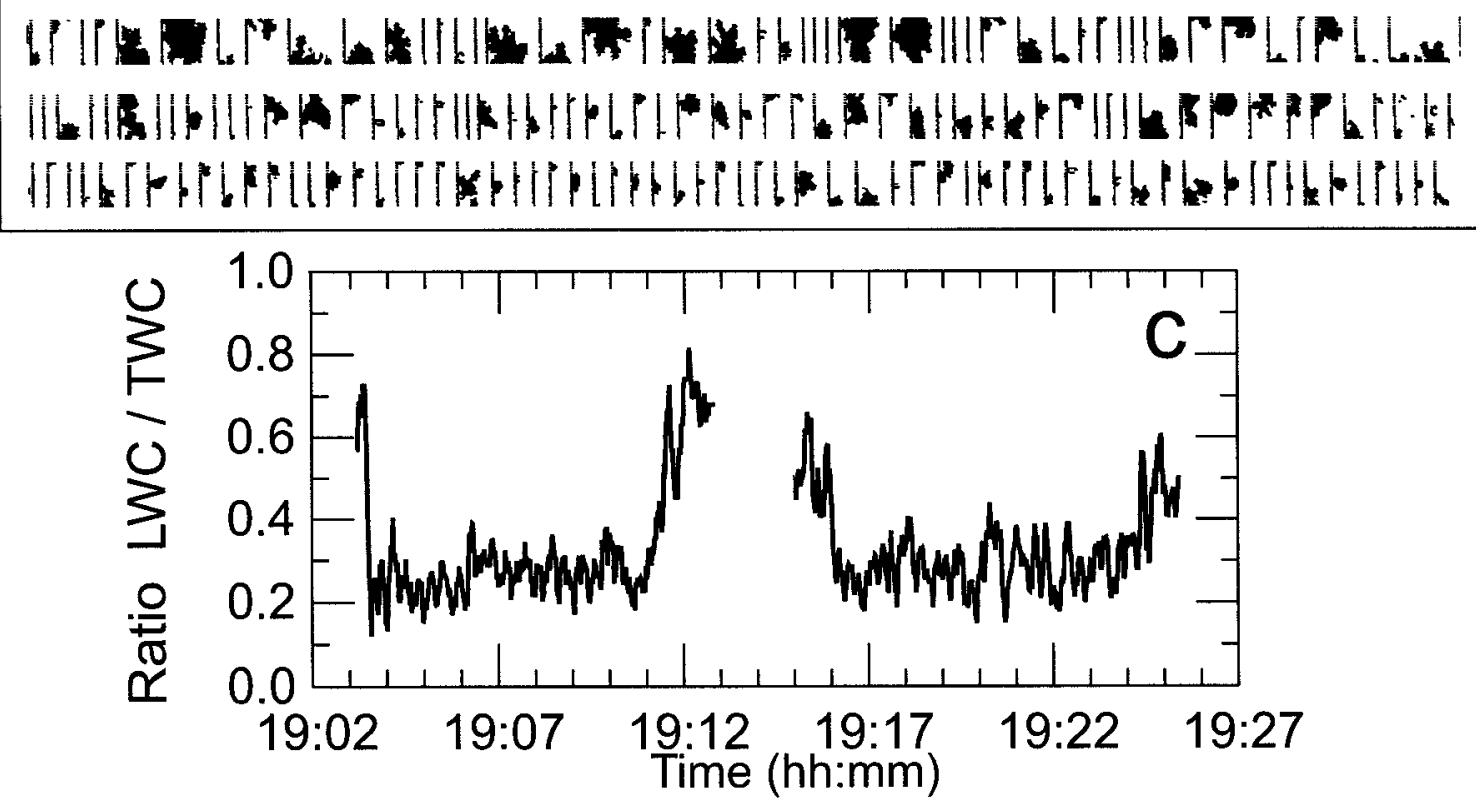

FIG. 15. (a) Time histories of the Nevzorov LWC and TWC probes in a mixed cumulus congestus on 19 August 1994, Ottawa: $H=6700$ $\mathrm{m} ; T=-15^{\circ} \mathrm{C}$. (b) OAP-2D-C images from the central part of the cloud. (c) The time history of the phase ratio LWC to TWC.

zorov LWC, TWC, and PMS King probes is usually within $10 \%$ and does not exceed $15 \%$ on average. We accept the results of these intercomparisons as a general estimate of accuracy of LWC measurements of the Nevzorov probe. The Nevzorov probe sometimes shows distinct advantages in low-LWC situations due to its superior baseline stability. Wind tunnel tests have established a good accuracy $(10 \%-20 \%)$ for the phase separation capability of the probe for small frozen droplets.
Examples of natural mixed-phase clouds reveal the probe's capability to instantly identify neighboring regions of glaciated and liquid-only cloud, consistent with the data from other instruments, but quantitative verification of the IWC measurement in natural snow and ice particles has not yet been possible due to the absence of any accurate reference standards. Further research is required to characterize the residual effect of ice particles on the LWC measurement. The TWC 

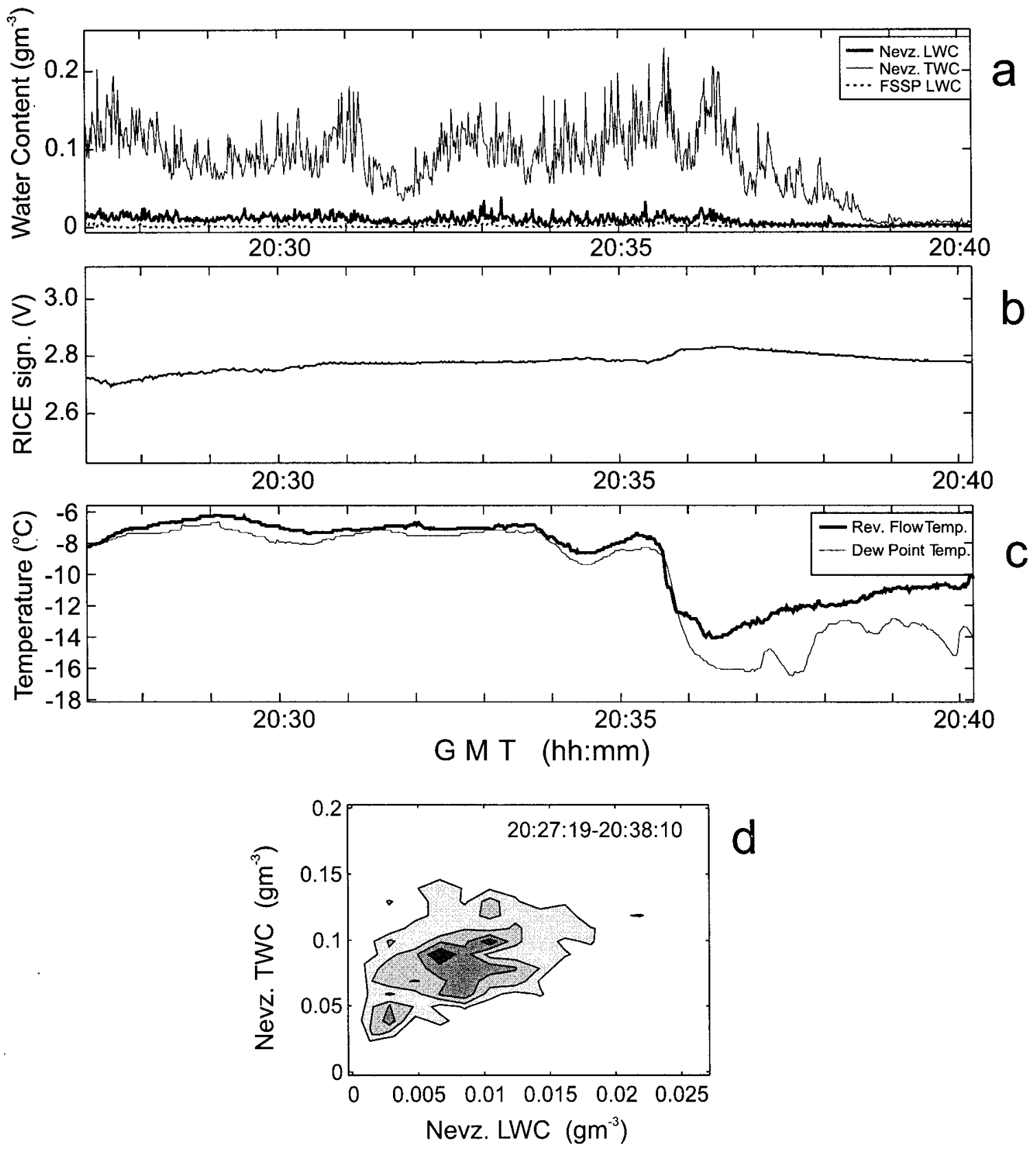

FIG. 16. Residual effect on the LWC sensor due to ice crystals. (a) Time histories of the Nevzorov LWC and TWC [and the FSSP (2-32 $\mu \mathrm{m})$ ] in glaciated cloud, 3 March 1995, Newfoundland: $H=2200-500 \mathrm{~m} ; T=-6^{\circ}--15^{\circ} \mathrm{C}$. (b) Time history of the RICE response; (c) time histories of the reverse flow and dewpoint (EG\&G 130) temperatures; (d) density scattergram of Nevzorov LWC and TWC for the data of (a). Each successive level of gray denotes the next $20 \%$ density level.

measurement offers the additional possibility of greatly improved liquid water contents in drizzle situations (when conventional hot-wire devices are known to underestimate) and, perhaps, water content measurements in light rain situations.

Acknowledgments. The authors express gratitude to Dr. Stewart Cober for help in the data analysis and the calculation of LWC derived from PMS probes. Special thanks to Phil Brown and to two reviewers for useful remarks and comments.

\section{REFERENCES}

Biter, C. J., J. E. Dye, D. Huffman, and W. D. King, 1987: The drop response of the CSIRO liquid water content. J. Atmos. Oceanic Technol., 4, 359-367.

Brown, P. R. A., 1993: Measurements of the ice water content in 
cirrus using an evaporative technique. J. Atmos. Oceanic Technol., 10, 579-590.

Heymsfield, A. J., and L. M. Miloshevich, 1989: Evaluation of liquid water measuring instruments in cold cloud sampled during FIRE. J. Atmos. Oceanic Technol., 6, 378-388.

King, W. D., D. A. Parkin, and R. J. Handsworth, 1978: A hot-wire water device having fully calculable response characteristics. $J$. Appl. Meteor., 17, 1809-1813.

— C. T. Maher, and G. A. Hepburn, 1981: Further performance test on the CSIRO liquid water content. J. Appl. Meteor., 20, 195-202.

Kosarev, A. L., I. P. Mazin, A. N. Nevzorov, and V. F. Shugaev, 1976 Optical density of clouds (in Russian). Trans. Cent. Aerological Observatory, 124, 33-43.

Nevzorov, A. N., 1980: Aircraft cloud water content meter. Comm. a la 8ieme Conf. Int. sur la Phys. des Nuages, Vol. 2, Clermont-
Ferrand, France, Int. Commission on Clouds and Precipitation, 701-703.

_ 1983: Aircraft cloud water content meter (in Russian). Trans. Cent. Aerological Observatory (Trudi TsAO), 19-26.

— ice phase evolution in supercooled clouds. Sov. Meteor. Hydro., N1, 69-76.

- and - 1992b: Experimental studies of phase-disperse structure of stratiform clouds at negative temperatures. Sov. Meteor. Hydro., N8, 41-51.

Twohy, C. H., A. J. Schanot, and W. A. Cooper, 1997: Measurement of condensed water content and ice clouds using an airborne counterflow virtual impactor. J. Atmos. Oceanic Technol., 14, 197-202.

Voloshchuk, V. M., 1971: The Introduction to the Hydrodynamics of Coarse-Dispersed Aerosols (in Russian). Gidrometeoizdat, 208 pp. 